\title{
FCSOFM Technique for Medical Image Segmentation
}

\author{
Anamika Ahirwar ${ }^{1}$ and R.S. Jadon ${ }^{2}$ \\ ${ }^{1}$ Department of Computer Science \& Engineering, Maharana Pratap \\ College of Technology, Gwalior \\ E-mail: aanamika77@gmail.com \\ ${ }^{2}$ Department of Computer Applications, Madhav Institute of Technology \\ \& Science, Gwalior \\ E-mail: rsj_mits@yahoo.com
}

\begin{abstract}
Medical image segmentation is a challenging task. This paper proposed FCSOFM a novel technique for medical image segmentation. FCSOFM technique segments the defected region of brain MRI and digital mammogram images. Then we calculate the efficiency of our proposed technique by the computation of confusion matrix.
\end{abstract}

Keywords: FCSOFM (Fuzzy C-means Self Organizing Feature Map), confusion matrix, membership matrix.

\section{Introduction}

In the field of medical imaging like detection of brain tumor, breast cancer, skin cancer, cervical cancer are the major problem now a days. If we diagnose the problem in early stages, doctors can easily cure it by given proper treatment to patients. Today a very critical and serious problem can be seen among children and adults is Brain tumor[14,15]. Brain tumor an abnormal growth of cells within the brain. Breast cancer the second main cause of cancer deaths in women's is also a very serious problem. The death rate can be reduced if it detects at its early stages. According to the report of United States, in 2014 estimated new cases and deaths from breast cancer are 235,030 [2,360(male), 232,670(female)] and 40,430 [430(male), 40,000(female)] respectively[2], from brain and other nervous system cancers are 23,380 and 14,320 respectively[18] and from cervical cancer are 12,360 and 4,020 respectively[1]. Over the past period, a variety of techniques has been proposed for automated segmentation of medical images, with different levels of computerization and practical applicability. Most of the methods involve clustering training samples and relating clusters to given categories. The complexity and limitations of these mechanisms are mainly due to the lack of an effective way to state the boundaries between clusters. This problem becomes more obstinate as the number of features used for classification increases. On the opposite, the fuzzy classification predicts that the boundary between the two neighboring classes to be a continuous and overlapping area within which an object has partial membership of each class. This perception reflects the reality of many applications in which categories have poorly defined boundaries and also provides a simple illustration of the complex partition of the data space. Authors proposed various techniques for the automation of tumor/cancer detection in medical image. Rohan Kandwal et al.[6] proposed an approach to detect and segment brain tumor. Rohini Paul Joseph et al.[5] proposed a segmentation technique using K-means clustering method followed by morphological filtering which avoids the misclustered regions of brain MRI image that can certainly be formed after segmentation for finding the location of tumor. Roshan G.Selkar et al.[3] used Watershed and Thresholding method to detect brain tumor, boundary of tumor and size of tumor. To extract watershed lines from a topographic representation of the input breast cancer image, Sura Ramzi Shareef[4] proposed a segmentation method which is based on morphological watershed transform. Apart from 
these various techniques like watershed[8,9], artificial neural network[10], fuzzy and wavelet technique[7], decision tree ID3[11], k-NN and fuzzy c-means[12], BackPropagation Neural (BPN)[13], and many more are used for analysis, classification and segmentation of medical imaging.

Rest of the sections is described as follows; section II describes the brief overview of FCSOFM (Fuzzy C-means Self Organizing Feature Map) technique used to segment the affected region in medical imaging. In section III, results of medical image segmentation and their properties are shown. Finally we conclude in section IV.

\section{FCSOFM (Fuzzy C-means Self Organizing Feature Map) Technique}

FCSOFM technique is basically a hybrid technique of fuzzy c-means and self organizing feature map approach. Many authors seperatly used fuzzy and self organizing feature map tehnique to detect the affected region of the input medical image. This unsupervised clustering technique is used to cluster the region of medical image. SOFM is an unsupervised clustering method which consists components called nodes. Firstly, pixels are clustered which is based on their grayscale value and spatial features, where the clustering process is effectuated with a Self Organizing Feature Map network. Clustering technique separates different regions. These regions could be considered as segmentation outcomes which have some semantic meaning. Every node of SOFM contains a corresponding same dimension weight vector. On every step of the learning process a random vector is chosen from the initial data set to identify the best matching neuron coefficient vector. Then after select the winner node which is most similar to the input vector. Then by Euclidean metric distance is measured between the vectors. Track smallest distance node between the vectors. This node is known as best matching unit. Then update nodes in the neighborhood of best matching unit by pulling them closer to the input vector. For fuzzy c-means algorithms the result of neighborhood function is an initial cluster center i.e. centroids. To find cluster centers we use Fuzzy c-means clustering method. The membership matrix is randomly initialized in order to assimilate the fuzzy partitioning technique. When the difference of update membership matrix and membership matrix is less than the termination criterion the iteration will stop and this termination criterion lies between 0 and 1 .

\section{Results}

We apply our proposed technique to test 50 brain MRI images but here we are showing the result of a normal and cancerous brain MRI image in Table 1 and Table 2 respectively. In Table 1 the input normal image is taken from the Keith's database[16], but according to our proposed technique it is an abnormal image. Very less number of pixels detected in the area of region so we can consider it as a no deformity in image. But our eyes cannot see this minor deformity in the image. The extraction of regions and their statistical properties for normal and cancerous brain MRI image are shown in Table 1 and Table 2 respectively. The proposed technique also applies on digital mammogram images. The mammogram database is taken from Digital Database for Screening Mammography(DDSM)[17]. In Table 3 and Table 4 we show the result of extracted tumor region and its statistical properties of normal and cancerous mammogram image respectively. After that we calculate the efficiency of our proposed scheme using confusion matrix. Confusion matrix is a picturing tool which consists of True Negative, False Positive, False Negative and True Positive. Each column of confusion matrix denotes the instances in a predicted class and each row denotes the instances in an actual class. We test 50 test cases of brain MRI images in which 32 cases are True Positive means our test says that they have positive results called as True Positives. Some have the tumor, but the test says they don't have tumor are called False Negatives. In our test there are 11 cases which are False Negative. Some cases don't have the tumor, and the test says they don't, they are True Negatives and our test shows there are 8 such cases. Finally we have some images which are normal brain MRI images who have a positive test result; 
these are False Positives which is the only one case in our test. False Negatives and False Positives are significant issues in medical testing. Similarly we check total 50 test cases of mammogram images in which 20 cases are True Positive, 10 False Positive, 18 False Negative and 02 True Negative. Table 5 shows the accuracy of the results of Brain MRI and Digital Mammogram images. The accuracy calculated for normal brain MRI images is $91.67 \%$ and cancerous images are $84.21 \%$. The accuracy of normal and cancerous mammogram images are $90 \%$ and $86.67 \%$ respectively.

\section{Conclusion}

This paper describes FCSOFM technique to segment the brain and digital mammogram image. FCSOFM technique extracts the cancerous region from the medical images. We work on noiseless medical images. We compare our tested results from the available databases on the web, and calculate the effectiveness of our proposed technique. Effectiveness can be calculated by applying statistical test with the help of confusion matrix.

\section{Table 1. Extraction of Regions and Their Statistical Properties of Normal Brain MRI Image}

\begin{tabular}{|c|c|c|c|c|c|c|}
\hline $\begin{array}{c}\text { Properties of } \\
\text { Extracted Region }\end{array}$ & White Matter & Gray Matter & CSF & Tumor & \multicolumn{2}{|c|}{$\begin{array}{c}\text { Properties of Tumor Region } \\
\text { (Tumor Detection) }\end{array}$} \\
\hline & & & & & Energy & $\begin{array}{l}1.9963773778656 \\
764 \mathrm{E}-9\end{array}$ \\
\hline Name of region & White matter & Gray matter & CSF & Tumor & Entropy & $\begin{array}{l}-0.001956010686 \\
6928974\end{array}$ \\
\hline Type of region & Medium & Medium & Medium & Small & IDM & $\begin{array}{l}3.8103947568968 \\
156 \mathrm{E}-5\end{array}$ \\
\hline $\begin{array}{c}\text { Average grayscale } \\
\text { value of region }\end{array}$ & 48.0 & 48.0 & 46.0 & 270.0 & Contrast & $\begin{array}{l}1.7146776406036 \\
663 \mathrm{E}-4\end{array}$ \\
\hline $\begin{array}{l}\text { Area of region } \\
\text { (pixels) }\end{array}$ & 13128 & 7029 & 13123 & 13 & Mean & $\begin{array}{l}1.7146776406045 \\
664 \mathrm{E}-4\end{array}$ \\
\hline Centroid of region & - & - & - & 121,167 & $\begin{array}{l}\text { Standard } \\
\text { deviation }\end{array}$ & $\begin{array}{l}1.9567372721454 \\
357 \mathrm{E}-5\end{array}$ \\
\hline
\end{tabular}

Table 2. Extraction of Regions and Their Statistical Properties for Cancerous Brain MRI Image

\begin{tabular}{|c|c|c|c|c|c|c|}
\hline $\begin{array}{c}\text { Properties of } \\
\text { Extracted Region }\end{array}$ & White Matter & Gray Matter & CSF & Tumor & \multicolumn{2}{|c|}{$\begin{array}{c}\text { Properties of Tumor Region } \\
\text { (Tumor Detection) }\end{array}$} \\
\hline & & & & & Energy & $\begin{array}{l}1.1960093386154 \\
378 \mathrm{E}-11\end{array}$ \\
\hline Name of region & White matter & Gray matter & CSF & Tumor & Entropy & $\begin{array}{l}-2.618446108420 \\
8006 \mathrm{E}-4\end{array}$ \\
\hline Type of region & Medium & Medium & Medium & Small & IDM & $\begin{array}{l}2.8775101480191 \\
317 \mathrm{E}-6\end{array}$ \\
\hline $\begin{array}{c}\text { Average grayscale } \\
\text { value of region }\end{array}$ & 34.0 & 29.0 & 32.0 & 51.0 & Contrast & $\begin{array}{l}1.8224230937453 \\
446 \mathrm{E}-5\end{array}$ \\
\hline $\begin{array}{l}\text { Area of region } \\
\text { (pixels) }\end{array}$ & 18457 & 10009 & 18450 & 501 & Mean & $\begin{array}{l}1.8224230937454 \\
443 \mathrm{E}-5\end{array}$ \\
\hline Centroid of region & - & - & - & 200,154 & $\begin{array}{l}\text { Standard } \\
\text { deviation }\end{array}$ & $\begin{array}{l}7.1916007577212 \\
77 \mathrm{E}-7\end{array}$ \\
\hline
\end{tabular}




\section{Table 3. Extraction of Regions and Their Statistical Properties of Normal Digital Mammogram Image}

\begin{tabular}{|c|c|c|c|c|c|}
\hline Input Image & Result & \multicolumn{2}{|c|}{ Properties of Extracted Region } & \multicolumn{2}{|c|}{$\begin{array}{c}\text { Properties of Tumor Region } \\
\text { (Tumor Detection) }\end{array}$} \\
\hline & 4hore & Type of region & Small & Energy & $4.6100467443467187 \mathrm{E}-8$ \\
\hline & & Average gray scale value of region & 0.0 & Entropy & -0.0021438427064320744 \\
\hline & & Area of region (pixels) & -39 & IDM & $2.288818359373 \mathrm{E}-4$ \\
\hline & & Centroid of region & 8,8 & Contrast & $2.44140626 \mathrm{E}-4$ \\
\hline & & & & Mean & $2.44140624 \mathrm{E}-4$ \\
\hline Normal & Normal & & & $\begin{array}{l}\text { Standard } \\
\text { deviation }\end{array}$ & $5.623722714419875 \mathrm{E}-6$ \\
\hline
\end{tabular}

Table 4. Extraction of Tumor Region and Their Statistical Properties of Cancerous Digital Mammograms Image

\begin{tabular}{|c|c|c|c|c|c|}
\hline Input Image & Result & \multicolumn{2}{|c|}{ Properties of Extracted Region } & \multicolumn{2}{|c|}{$\begin{array}{c}\text { Properties of Tumor Region } \\
\text { (Tumor Detection) }\end{array}$} \\
\hline & & Type of region & Small & Energy & $1.4365800608474146 \mathrm{E}-11$ \\
\hline & & Average gray scale value of region & 88.0 & Entropy & $-1.4474218793470595 \mathrm{E}-4$ \\
\hline & & Area of region (pixels) & 907 & IDM & $1.0273691131749825 \mathrm{E}-5$ \\
\hline & & Centroid of region & 106,234 & Contrast & $1.0273691131749814 \mathrm{E}-5$ \\
\hline & & & & Mean & $1.0273691131749813 \mathrm{E}-5$ \\
\hline Cancer & Cancer & & & $\begin{array}{l}\text { Standard } \\
\text { deviation }\end{array}$ & $1.7466296110876375 \mathrm{E}-7$ \\
\hline
\end{tabular}

Table 5. Result of Accuracy of Brain MRI AND Digital Mammogram Images

\begin{tabular}{|l|l|l|l|l|l|}
\hline \multicolumn{1}{|c|}{ Image } & $\begin{array}{c}\text { Total tested } \\
\text { cases }\end{array}$ & \multicolumn{1}{|c|}{ Type of image } & $\begin{array}{c}\text { Total images } \\
\text { (expected) }\end{array}$ & $\begin{array}{c}\text { Total images } \\
\text { (observed) }\end{array}$ & Accuracy \\
\hline \multirow{2}{*}{$\begin{array}{l}\text { Brain MRI } \\
\text { image }\end{array}$} & \multirow{2}{*}{50} & Normal images & 12 & 11 & $91.67 \%$ \\
\cline { 2 - 6 } $\begin{array}{l}\text { Mammogram } \\
\text { image }\end{array}$ & \multirow{2}{*}{50} & Cancerous images & 38 & 32 & $84.21 \%$ \\
\cline { 3 - 6 } & & Normal images & 20 & 18 & $90.00 \%$ \\
\cline { 2 - 6 } & Cancerous images & 30 & 26 & $86.67 \%$ \\
\hline
\end{tabular}

\section{References}

[1] http://www.cancer.gov/cancertopics/types/cervical.

[2] "American Cancer Society, Cancer Facts \& Figures", Atlanta: American Cancer Society, (2014).

[3] R. G. Selkar, et al., "Brain Tumor Detection and Segmentation by Using Thresholding and Watershed Algorithm", International Journal of Advanced Information and Communication Technology, ISSN 2348 - 9928, vol.1, no.3, (2014), pp.321-324.

[4] S. R. Shareef, "Breast Cancer Detection Based on Watershed Transformation", International Journal of Computer Science Issues, ISSN (Print): 1694-0814 | ISSN (Online): 1694-0784, vol.11, no.1, (2014), pp.237-245.

[5] R. P. Joseph, et al., "Brain Tumor MRI Image Segmentation and Detection in Image Processing", International Journal of Research in Engineering and Technology, eISSN 2319-1163 | pISSN 23217308, vol.3 no.1, (2014), pp 1-5.

[6] R. Kandwal, et al., "An Automated System for Brain Tumor Detection and Segmentation”, International Journal of Advanced Research in Computer Science and Software Engineering, ISSN 2277 128X, vol.4, no.3, (2014), pp.97-100.

[7] N. S. Ramteke, et al., "Analysis of Skin Cancer Using Fuzzy and Wavelet Technique - Review", International Journal of Engineering Trends and Technology (IJETT), vol.4, no.6, (2013), pp.25552566.

[8] S. L. Kadachha and P. J. Patel, "Cancer Detection Using Modified Watershed", International Journal of Engineering Research \& Technology, vol.2 no.5, (2013), pp.2161- 2164.

[9] B. K. Saptalakar et al., "Segmentation Based Detection of Brain Tumour", International Journal of Computer and Electronics Research, vol.2, no.1, (2013), pp.20-23. 
[10] J. A. Jaleel, et al., “Artificial Neural Network Based Detection of Skin Cancer”, International Journal of Advanced Research in Electrical, Electronics and Instrumentation Engineering, vol.1, no.3, (2012).

[11] S. P. Angayarkanni, et al., "Efficient Classification of Cancerous Masses in Mammogram", International Journal of Mathematics and Computer Applications Research, ISSN 2249-6955, vol.2, no.3, (2012), pp.10- 26.

[12] T. C. Cahoon, "Breast Cancer Detection Using Processing Techniques", Fuzzy Systems, Ninth IEEE International Conference, ISSN No. 1098-7584, ISBN No. 0-7803-5877-5, vol.2, (2000), pp.973-976.

[13] M. Antonie and et al., "Application of Data Mining Techniques for Medical Image Classification", In Proc., Second International Workshop on Multimedia Data Mining (MDM/KDD) in Conjunction with ACM SIGKDD, (2001); San Francisco, USA.

[14] http://www.braintumourindia.com/BTF/braintumours.htm.

[15] http://www.braintumor.org.

[16] K. A. Johnson and J. Alex Becker, "The whole brain Atlas",

[17] University of South Florida Digital Mammography Home Page.

[18] http://www.cancer.gov/cancertopics/types/brain. 
International Journal of Bio-Science and Bio-Technology

Vol.7, No.4 (2015) 